\title{
Dr Junkie. The Doctor Addict in Bulgakov's Morphine: What are the Lessons for Contemporary Medical Practice?
}

\author{
Victoria Tischler
}

Published online: 13 November 2013

(C) The Author(s) 2013. This article is published with open access at Springerlink.com

\begin{abstract}
Historical, cultural and professional factors have contributed to stigma and secrecy regarding addiction in the medical profession and there are calls to improve education in this area. This paper argues that physician-penned literature plays an important role in raising awareness of substance misuse in the medical profession. Bulgakov's short story Morphine documents the decline of Dr Polyakov and illustrates a number of salient professional issues such as self-medication, abuse of authority and risks to patients. Physician-penned literature such as Morphine is of value in medical education as it offers a route into sensitive topics and an authoritative and insider perspective that is attractive to students and physicians alike.
\end{abstract}

Keywords Addiction $\cdot$ Medical education $\cdot$ Opiates $\cdot$ Physicians $\cdot$ Professionalism

\section{Background}

The use of opium and its derivatives to alter consciousness and relieve pain has been reported since ancient times (Foxcroft 2007; Burton 2006; Porter and Teich 1998). The Sumerian civilisation are understood to have cultivated the opium poppy or 'joy plant' in lower Mesopotamia (now Iraq) as far back as 4000-3400BC (Santella 2007; Foxcroft 2007), the Egyptian medical text the Ebers Papyrus dating from 1600BC describes the poppy plant in detail (Jay 2010), and in the Byzantine period opium was taken as an elixir (Ramoutsaki et al. 2002).

Physicians have a longstanding relationship with the preparation and use of opiates. The 17th Century physician Thomas Sydenham's bestselling laudanum mixture contained two ounces of opium in port or wine, spiced with saffron, cinnamon and cloves and was labelled 'Laudanum Sydenhamii'. 'Dover's Powder', invented by physician Thomas Dover in 1732 combined opium powder, liquorice and the emetic ipecacuana (Jay 2010). By the early 19th Century opium use was commonplace. It was sold in pill or tincture form by a varied group of traders including apothecaries, tailors, bakers and rent collectors (Foxcroft 2007). The substance was used widely by the public for self-medication in a range of ailments including menstrual pain, intestinal pain and psychological disorders.

V. Tischler $(\bowtie)$

Division of Psychiatry and Applied Psychology, Institute of Mental Health, University of Nottingham, Triumph Road, Nottingham NG7 2TU, UK

e-mail: victoria.tischler@nottingham.ac.uk 
Self-experimentation is common in the history of science and pharmacology is no exception. In 1817 morphine was derived from opium by a German pharmacist Freidrich W. Sertürner after a series of experiments on himself and others (Jay 2010). This breakthrough heralded the availability of a substance, named after Morpheus the Greek god of dreams, which was of a predictable concentration thus increasing its medicinal value yet making it more expensive and decreasing its availability to individuals for self-medication. By 1850, with the advent of the hypodermic syringe, the therapeutic use of morphine became widespread, becoming known as the 'silver bullet'(Foxcroft 2007). This was due to its efficacy in managing pain, a reputation which remains to the current day.

\section{Physicians' use of opium}

Reports of opiate use in the medical profession tend to focus on experimental usage or selfmedication, a stance that may be construed as ambivalence particularly if physicians are involved in research involving drug-testing and are assumed to have substantial knowledge of pharmacology. The 'self-treatment' practised by physicians is unique to them as a population given their expertise and training, their key role in drug administration, and their access to controlled drugs. Despite advice to the contrary, many physicians continue to selfprescribe (British Medical Association-BMA, 2012; Hem et al. 2005; Bennett and O'Donovan 2001; Montgomery et al. 2011) presenting clear risks to the individual and to patients in their care (Brooks et al. 2011a). The tradition of 'self-experimentation' where clinicians used their bodies as 'laboratories' has been well-documented, for example Sigmund Freud's use of cocaine which he described as a 'magical drug' and which was later used as a local anaesthetic, Thomas Beddoes, the physician who discovered the medicinal utility of nitrous oxide, and the surgeon William Stuart Halstead who experimented with cocaine and morphine (Imber 2011; Jay 2010).

One 'self-experimenter' Oscar Jennings documented his personal use of morphine. Jennings was a physician admitted to the Royal College of Surgeons in the UK in 1873 . He was an expert on spinal disease who also wrote several monographs on 'the morphine treatment'. His language suggests an empathic stance with his patients, for example, he asserted that we 'believe the expert' and there is coy reference to his own drug use, for example 'my first observations were made in my own case' (Milligan 2005). Such a testimonial highlights the characteristics of physician-users which may be problematic, in particular a sense of scientific authority masking substance misuse.

\section{Addicted physicians}

Early reports suggest that substance misuse can be identified early in medical training. Sir James Paget, a British surgeon and pathologist studied medical students during the period 1839-1859 and noted that, of the 56 of 1000 that failed their exams, 10 (17.8\%) did so because of drug or alcohol problems 'through the continuance in habits of intemperance or dissipation as had made us, even when they were students, anticipate their failure' (Marshall 2008). Other historical accounts suggest that habitual use of morphine in the profession was relatively common. The US physician J. B. Mattison made the startling claim in 1883 that 'most morphine habitués were doctors', citing rates of addiction in doctors of 30-40\% (Milligan 2005). In 1909 Oscar Jennings claimed that morphinism was responsible for one fifth of all deaths in the medical profession (Milligan 2005). In 1924 a German 
psychopharmacologist Louis Lewin suggested that it was not just $40 \%$ of doctors but also $10 \%$ of their wives who were addicted to morphine (Milligan 2005; Jay 2010).

More recent research suggests that substance misuse in the medical profession is related to a combination of occupational and individual factors including job stress, availability of medication, maladaptive coping, and self-critical tendencies (Brooks et al. 2011a). Much of the relevant literature focuses on alcohol dependence which at $13-14 \%$, is similar to rates in the general population (Hughes et al. 1992) although it is acknowledged that the 'hard drinking' culture in medical school persists (Ritson 2001; Wallace 2000). There are also particular risks regarding misuse of illicit drugs in this population including compromised patient care and higher rates of completed suicide compared to the general population (Schernhammer and Colditz 2004). The media has raised the issue with high profile cases involving abuse of patients including Harold Shipman, a British General Practitioner (family physician) who was convicted of murdering his elderly patients and who was dependent on pethidine (British Broadcasting Corporation-BBC 2004).

Prescription drug misuse particularly of opiates and other analgesics, is higher in physicians (Hughes et al. 1992) and medical students (Westermeyer 1991) compared to similar populations as is a reluctance to seek help (Merlo and Gold 2008). As Boisaubin and Levine (2001) suggest, some of the characteristics that make physicians successful in clinical practice such as conscientiousness, a drive to achieve, and denial of personal issues can also make them harder to help if their performance is impaired. Some specialities such as anaesthesiology are at particular risk of substance misuse (Tetzlaff et al. 2010) and junior physicians are especially vulnerable (Brooks et al. 2011a). Anaesthesiologists and surgeons may be at risk of addiction due to sensitisation through inhalation of drugs such as the synthetic opiate fentanyl and propofol, a hypnotic sedative, in surgical environments (McAuliffe et al. 2006). A report by the Practitioner Health Programme (PHP) set up in the UK to treat impaired physicians suggested that they were addicted to 'every drug under the sun' (Ramesh 2010) including heroin, methadone, amphetamines and ketamine and that of the almost $40 \%$ of referrals who presented with addiction problems, $4.5 \%$ were addicted to opiates. It is suggested that problems are often severe by the time that treatment is sought (Brooks et al. 2011b). This is unsurprising as sanctions for transgression include suspension or ban from medical practice, factors which will inhibit identification and help-seeking in this population. Substance misuse and mental health problems can be identified in more than $30 \%$ of individuals before they begin medical studies (Fletcher and Ronis 2005) suggesting that early provision of information about substance misuse during training may be beneficial.

\section{Raising awareness of the issue}

A recent report notes that medical students are reluctant to seek help for mental health difficulties including drug and alcohol abuse as they are fearful of potential negative repercussions for their career (Grant et al., 2013). Many students are unaware of service provision in this area whilst at medical school according to the British Medical Association's 2007 welfare survey (email to the author, June 10, 2013). Support systems in medical schools are recommended to combat the stress and pressure which may lead some to abuse substances (Jones 1993; Stecker 2004). Teaching on substance misuse overall is viewed critically by medical students (Brown 2006) and is often an 'add on' for example to a psychiatry rotation (Yoast et al. 2008) with students developing stigmatised attitudes towards people who abuse drugs and alcohol early in their training (Rasyidi et al. 2012). A report of the International Centre for Drug Policy (ICDP 2007) working party recommended that raising awareness of 
substance misuse by medical students and their colleagues should be a core educational aim, so that the impact on their own health and behaviour and professional conduct may be recognised and so that impaired performance in colleagues can be identified and responded to effectively. In particular, topics such as professionalism, fitness to practice, ethics and stigma need further development (Notley et al. 2013; Royal College of Psychiatrists 2011).

There is very little in the way of teaching materials specific to physician misuse of substances. First person accounts by physician-addicts are sparse given the stigma associated with addiction however a number of examples exist. These include pleas for addiction to be considered a disease and warning against the prescribing of painkillers to colleagues in recovery from addiction (National Institute on Drug Abuse-NIDA 2013). Other websites targeting physicians such as the British Doctors and Dentists group (BDDG) and social media site KevinMD post such accounts see 'the drugs' (BDDG, 2013) and 'a physician story of addiction and recovery' (Anon, 2010). Such testimonials are by their nature idiosyncratic and therefore are not always useful for teaching. In particular problematic professional issues regarding procuring drugs, attendant risks to clinical practice, and how colleagues may usefully respond are not presented. Instead the above accounts detail the personal consequences of drug misuse such as emotional and marital problems and practical issues about rehabilitation in the form of so-called recovery narratives (McIntosh and McKeganey 2000).

\section{A role for the humanities?}

A variety of humanities resources have been recommended to teach medical students about substance misuse albeit this is not directly related to physician drug misuse. The use of interactive teaching methods to develop skills and confidence is recommended (Polydorou et al. 2008). It is suggested that film can be used to address some of the complexities related to addiction (Cape 2009) and a number of films are recommended in medical education for example 'Drunks' in which a female physician addicted to Demerol (pethidine) engages in a 12-step (Alcoholics Anonymous) recovery programme. This film may be used to stimulate discussion about impaired colleagues, addiction to substances and work addiction, and how these issues may be dealt with by colleagues (Alexander et al. 2006). The National Institute on Drug Abuse (NIDA) has established teaching materials for medical students and physicians in training (NIDAMED) including a theatre project which aims to trigger empathic responses in the viewers, enabling them to identify with the addict on an emotional level (NIDA 2013).

The use of literature is recommended in physician education to stimulate holistic practice and to promote empathy (Tischler 2010). Literature facilitates the vicarious experiencing of characters' lives therefore using first person accounts of addiction will help facilitate empathy (McNaughton 2000). Physician-penned writing can attract readers to the profession of medicine (Verghese 2010) and helps to foster self-reflection (Chopra 2010), a skill which is highly valued in the development of medical professionalism. The perspective of a physicianaddict lends authenticity to the account, a factor which medical students state to be important (Oyebode, 2013) and physician-writers may act as positive role models to encourage creativity in students and attract them to particular specialities (Tischler et al. 2011). Physician accounts also may assist with consideration of ethical dilemmas concerning colleagues (Jiten Mistry, personal communication June 26 2013).

The story Morphine is one of few first person accounts of physician addiction written by a physician-author. It illustrates many salient features of substance misuse in this population in the context of clinical practice. As a semi-autobiographical tale the writer speaks from an insider perspective and from a position of authority as the author Bulgakov was himself a physician. 


\section{Bulgakov and Morphine}

Mikhail Bulgakov was born in 1891 in Kiev, Ukraine. He graduated as a physician from Kiev University in 1916. He saw active service in the First World War as a field doctor and worked for 18 months in Smolensk in a remote country hospital. It is thought to be his experiences there that inspired the semi-autobiographical writings in A Country Doctor's Notebook (Bulgakov 1975), a collection of short stories in which Morphine appears. In 1920 he gave up medical practice to focus on his writing career. During the period 1925-1927 he worked as a freelance journalist and published short stories. He wrote for the lay press and for medical periodicals e.g. Meditisinsky Rabotnik (The Medical Worker). He experienced censorship under Stalin and many of his works were not published until after his death in 1940. His masterpiece The Master and Margarita was published in 1938.

The themes from A Country Doctors Notebook continue to resonate with residents today. They include fear of responsibility for others' health and well-being, lack of confidence due to inexperience, and loneliness and boredom in regard to routine and mundane tasks. Bulgakov establishes the status and superior authority of the physician in his text by contrasting the 'electric lights' of the city which he associates with intellectual pursuits, with the darkness of the rural area in which he is based and practising as a resident. This lends emphasis to the elite position of the physician. Hospitals and health care environments remain hierarchical environments with physicians typically assuming leadership roles and residents reluctant to challenge authority (Lerner 2007).

Bulgakov became addicted to morphine after he began to treat himself for chronic abdominal pain, apparently following his wounding during active service. Other reports indicate that Bulgakov contracted diphtheria from a child patient during a tracheotomy. This operation is described in the story of The steel windpipe, also from A Country Doctors Notebook.

The story Morphine is about Sergei Polyakov, a resident who becomes addicted to morphine whilst working in a remote rural hospital. The narrative unfolds through Polyakov's diary entries. He begins using morphine to treat physical pain, the first dose being administered by Anna Kirillovna, a married nurse with whom he begins a doomed affair. As his use of morphine increases, he notes its efficacy, not just for analgesia but also in dulling the emotional distress he experiences after his lover, an opera singer leaves him. As his addiction deepens, the tale reveals various justifications and rationalisations for his continued usage. His account echoes the sentiments of the 'self-experimenters' as he suggests that he is testing morphine to appreciate its therapeutic value, 'I must give due praise to the man who first extracted morphine from poppyheads. He was a true benefactor of mankind. The pain stopped 7 min after the injection' (p. 125) and, in justifying his usage, 'It would be a good thing if a doctor were able to test many drugs on himself. He would then have a completely different understanding of their effect' (p. 125)

The propensity for physicians to self-medicate is noted as Polyakov attempts to manage his symptoms using detached and scientific language, 'The pain came again...fearing a recurrence of yesterday's attack, I injected myself in the thigh with one centigramme [of morphine]. The pain ceased almost instantaneously'(pp 125-126).

As Polyakov's addiction takes hold he begins to manipulate others, in particular Anna, and abuses his professional position, in order to maintain his drug supply, 'Kindly give me the keys to the dispensary, I'm speaking as a doctor' (p. 129) and 'Are you going to do it [make up morphine solution]?' She... answered quietly 'Alright, I'll do it' (p. 129).

Collusion and denial within the medical profession represent cultural factors which can create barriers to help-seeking for the addicted physician. This can be seen in Polyakov's exchange with his treating physician (Doctor $\mathrm{N}$ ) in a Moscow rehabilitation clinic. Doctor $\mathrm{N}$ is 
persuaded by the addicted Polyakov to discharge him and is dissuaded from reporting him to the authorities. Doctor N states: 'Strictly speaking my dear fellow, you are not fit to practise and I shall be breaking the law if I do not notify your local medical authority to that effect...' to which Polyakov replies: 'I beg you Professor...not to tell them [local medical authority] anything...I'd be struck off with ignominy for being an addict... surely you wouldn't do that to me?' (p. 135).

The risk to patients is revealed as Polyakov minimises his use of morphine. He states: 'In fact there is nothing unusual or alarming about my condition. It does not in the least affect my capacity to work...I cope splendidly with operations, I am irreproachably careful when prescribing' (p. 131-132) and 'surely mild habituation is not the same as becoming an addict?' (p. 130). Like Oscar Jennings, Polyakov expresses empathy with patients, 'Having suffered from this appalling malady, I hereby enjoin all doctors to be more compassionate towards their patients' (p. 133). This is evocative of a Jungian 'wounded healer' (Stevens 2001). That is, in acknowledging his own pain and vulnerability Polyakov becomes more empathic towards his patients. Whilst this stance may have some substance it also demonstrates the depth of Polyakov's denial of his addiction.

The privileged access physicians have to opiates is illustrated as Polyakov, his condition worsening, his behaviour compulsive, steals from the hospital supplies, 'I also stole some morphine from the hospital...the key was in the lock of the hospital's drug cabinet. Supposing it had not been. Would I have smashed open the cupboard? Would I? In all honesty? Yes, I would' (p. 136).

Even when he expresses insight into his drug dependence Polyakov continues to deny his problems. His embodied description of addiction evokes the conflict he experiences yet his arrogant stance minimises the clear risk to his patients, 'It is absolutely true that I am degenerating; the breakup of my moral personality has set in. But I can still work, and I am incapable of inflicting harm or wrong on a single one of my patients' (p. 136), '[I have] no trouble with my work. I avoid operating on the days when I am overcome by uncontrollable vomiting and retching' (p. 140).

The need for peers to be vigilant yet supportive is underlined by the condemnation Polyakov experiences from colleagues. This is likely to delay help-seeking and may precipitate a crisis before treatment is sought. 'I sensed contempt behind his pity...he is after all a psychiatrist and ought to realise that I am a sick man' (p. 134), 'I swore at the feldsher [physician's assistant]. He just laughed... he had come to report me' (p. $143)$.

Finally, desperate, and as he realises he is dying, Polyakov writes to Bomgard, a former colleague, who has moved back to the city to work. He begs for help but before Bomgard can reach him, Polyakov commits suicide. He leaves his diary with Bomgard as a chronicle of his addiction. Bomgard deliberates about whether to share the diary's contents and says 'I cannot say with any certainty whether they [Polyakov's diary entries] are instructive or useful though I believe they are... Should I publish the diary which was entrusted to me? I should. Here it is' (p. 144). This statement indicates the potential educational value of the text for others.

\section{How might this tale be useful?}

Gunderman (2010) suggests that physicians wear a white-coat of invulnerability and stories such as this have the ability to remove it. Bulgakov's tale provides a graphic first person account of opiate misuse in the medical profession. Despite being written more than 80 years 
ago the raw, honest and comprehensive portrayal is particularly compelling due to its semiautobiographical nature, detailing the implications for clinical practice and the threat of exposure which makes physicians vulnerable. Embodied tales of medical practice can work to evoke a sense of vulnerability which can help students appreciate the process of professional development and the physical and psychical nature of medical practice (Poirier 2006). Unlike other addiction tales which focus on recovery narratives for example the 'personal growth' or 'AA' stories (Day and Smith 2003) there is no redemption or happy ending in Bulgakov's tale. As Rudin et al. (1998) states literature provides 'a neutral yet attention-getting' mode of raising ethical issues. Evans (2009) suggests that literature provides a more holistic education, one which incorporates cultural, intellectual and spiritual issues including examination of one's own moral values and attitudes towards self-care such as those visibly evoked in Morphine.

In contrast to other narratives of physician addiction such as the portrayal of the resident David and his addiction to cocaine in Abraham Verghese's The Tennis Partner (1998), Polyakov's procurement of drugs and impairment at work are described in detail as are the interactions with colleagues such as Anna Kirillovna, his treating psychiatrist and the feldsher, all of whom play a role in enabling Polyakov's behaviour, challenging him, or condemning him. In The Tennis Partner David's impairment at work is not the subject of the text and his misuse of cocaine takes place largely away from his clinical practice thus the impairment of his professional skills is not fully revealed. The description of Old Doc Rivers from William Carlos Williams' The Doctor Stories (1987) reveals a physician who, though addicted to 'dope', is viewed as an almost mythical figure in the community in which he works in the US in the 20th Century. Rivers is viewed by his patients as either a saviour or as irredeemable. Williams' tale does not reveal River's source of drug supply or provide any resolution at its end, indeed the narrator, a colleague, maintains an ambivalent stance throughout. Whilst identifying some of the contradictions of addictive behaviour such as the ability to function normally at the same time as undertaking transgressive behaviours, the issue of how to help an impaired colleague is not considered. The Hungarian physician and author Géza Csáth wrote extensively about his addiction to opiates in 'The Diary of Géza Csáth' (2004), even detailing the dosage of each injection of morphine taken. Whilst his is a brutally honest and graphic depiction of addiction it is also an account of his sexually addictive and abusive behaviour and depressive illness. The diary documents his physical and mental decline, but does not detail the consequences of his addiction on his clinical practice, how the author obtains his supply of morphine, nor the reactions of his colleagues. It is particularly difficult to empathise with the author as his compulsive and sexually abusive behaviour towards others, many of whom are his patients, is unjustifiable. Csáth's short story 'the surgeon' (1983) focuses on an impaired physician albeit one addicted to alcohol. The story therefore does not deal with the highly stigmatised issue of opiate misuse so has little ability to raise issues about transgressive behaviour in this context.

Although physician-addicts share many characteristics with other substance misusers such as compulsive usage, physical dependence, and psychological craving, they face additional barriers to identification and effective treatment which are presented in Morphine. These include stigma and systemic barriers such as long working hours and cultural issues such as denial and the acceptance of self-treatment (Mansky 2011; Kay et al. 2008). Also highlighted in Morphine is the issue of concealment of emotion in order to contain or deny vulnerability which is a pertinent issue in the medical profession (Day and Smith 2003; Boisaubin and Levine 2001). Additionally the story contains several representative features which makes it compelling and evidence based (Steiner 2007) such as the focus on a young male physician at the start of his career, a population particularly at risk of substance abuse. 


\section{Conclusions}

Physician training is dominated by assimilation of factual information in the form of clinical skills and knowledge. Teaching on substance misuse requires improvement and should incorporate consideration of 'physician as patient' an area where literature can play an important role. There remains a need for efforts to remove the stigma and secrecy around addiction in the medical profession and to encourage medical students and physicians to seek help. Physician-penned literature can provide an accessible route into this sensitive topic as students respond to the authenticity and insider perspective of the accounts. Bulgakov's Morphine can be used to raise awareness of cultural, personal and clinical issues confronting the physician-addict and those at risk of substance misuse. Effective use of teaching materials such as this short story will help confront the stigma of physician substance misuse thus helping to improve patient care and increasing the likelihood of addicted physicians reaching out for assistance.

Acknowledgments With thanks to Professor Femi Oyebode, Rory O Connor, Dr John Pearson, and the British Doctors and Dentists Group, Dr Richard Knox, Dr Jiten Mistry, Jane Haywood at the Practitioner Health (PHP) Programme, Bonnie Milford at the Coalition On Physician Education in Substance Use Disorders (COPE), and colleagues at the psychopathological literature conference, University of Cadiz, 2011, the Association of Medical Humanities conference in Cork, 2012 and to $\mathrm{VC}$ for inspiration.

Open Access This article is distributed under the terms of the Creative Commons Attribution License which permits any use, distribution, and reproduction in any medium, provided the original author(s) and the source are credited.

\section{References}

Alexander, Matthew, Patricia Lenahan, P. and Anna Pavlo. Cinemeducation. A comprehensive guide to using film in medical education. Oxford: Radcliffe, 2006

Anonymous. A physician story of addiction and recovery" Accessed July 31, 2013 http://www.kevinmd.com/ blog/2010/12/physician-story-addiction-recovery.html

British Broadcasting Corporation-BBC. "Harold Shipman: The killer doctor" Accessed August 2, 2013 http:// news.bbc.co.uk/1/hi/uk/3391897.stm.

Bennett, Jennifer, and O'Donovan, Diarmuid. "Substance misuse by doctors, nurses and other healthcare workers." Current Opinion in Psychiatry no. 14 (3) (2001):195-199.

Boisaubin, Eugene V., and Levine, Ruth E. "Identifying and Assisting the Impaired Physician" American Journal of the Medical Sciences: 322 (1) (2001): 31-36

British Doctors and Dentists Group-BDDG. "The Drugs: one doctor's experience with drugs" Accessed July 29, $2013 \mathrm{http}: / / w w w . b d d g . o r g /$ latest-information/

British Medical Association-BMA. "Doctors continue to self-prescribe" Accessed August 2, 2013. http://bma. org.uk/news-views-analysis/news/2012/october/doctors-continue-to-selfprescribe

Brooks, Samantha. K., Gerada, Clare. and Chalder, Trudie. "Review of literature on the mental health of doctors: Are specialist services needed?" Journal of Mental Health 20 (2) (2011a):146-156.

Brooks, Samantha. K., Chalder, Trudie. and Gerada, Clare. "Doctors vulnerable to psychological distress and addictions: Treatment from the Practitioner Health Programme." Journal of Mental Health 20 (2) (2011b): 157-164.

Brown, Philip. J. "Substance Misuse in the Undergraduate Medical Curriculum. Project background: a survey of recent literature". Accessed August 1, 2013 http://www.sgul.ac.uk/research/projects/icdp/pdf/curr-devliterature-review-nov-2006.pdf

Bulgakov, Mikhail A Country Doctor's Notebook. London: Vintage, 1975

Burton, Neel L. Psychiatry. Oxford: Wiley-Blackwell, 2006.

Cape, Gavin. "Movies as a vehicle to teach addiction medicine" International Review of Psychiatry; 21(3) (2009): 213-217 
Chopra, Arun. "When art and medicine collide". In Mental Health, Psychiatry and the Arts: a tecahing handbook, edited by Victoria Tischler. Oxford: Radcliffe, 2010

Csáth, Géza. The diary of Géza Csáth. Budapest: Angelusz \& Gold, 2004

Csáth, Géza."The Surgeon". In Opium and Other Stories, edited by Marianna. D. Birnbaum. New York: Penguin, 1983

Day, Ed and Smith, Iain. "Literary and biographical perspectives on substance use" Archives in Psychiatric Treatment. 9 (2003):62-68

Evans, Martyn. "Roles for Literature in Medical education" In Mindreadings. Lituature and Psychiatry, edited by Femi Oyebode, 15-24. London: Royal College of Psychiatrists, 2009

Fletcher, Carol E., and Ronis David L. "Satisfaction of Impaired Health Care Professionals with Mandatory Treatment and Monitoring." Journal of Addictive Diseases no. 24 (3) (2005):61-75.

Foxcroft, Louise. The making of addiction: the use and abuse of opium in nineteenth century Britain Aldershot: Ashgate, 2007.

Grant, Andrew, Rix, Andrew, Mattick, Karen, Jones, Debbie and Winter, Peter. "Identifying good practice among medical schools in the support of students with mental health concerns". General Medical Council. Accessed August 1, $2013 \mathrm{http} / /$ www.gmc-uk.org/Identifying_good_practice_among_medcal_schools_in_the support_of_students_with_mental_health_concerns.pdf_52884825.pdf

Gunderman, Richard. "Professionalism guards against neglecting self". American Medical News. Accessed August 1, 2013 http://www.amednews.com/article/20100816/profession/308169947/5/\#top

Hem, Erlend, Stokke, Guro, Tyssen, Reidar, Grønvold, Nina T., Vaglum, Per. and Ekeberg, Øivind. "Selfprescribing among young Norwegian doctors: a nine-year follow-up study of a nationwide sample." $B M C$ Medicine 3 (1) (2005):16, accessed August 1, 2013. doi:10.1186/1741-7015-3-16

Hughes, Patrick H., Brandenburg, Nancy, DeWitt, C. Baldwin Jr, Storr, Carla L., Williams, Kristine. M., Anthony, James C. and Sheehan, David V. "Prevalence of Substance Use Among US Physicians." JAMA: The Journal of the American Medical Association no. 267 (17) (1992): 2333-2339. doi: 10.1001/jama.1992.03480170059029.

Imber, Gerald. Genius on the Edge: The Bizarre Double Life of Dr. William Stewart Halsted Kaplan Publishing, 2011.

International Centre for Drug Policy-ICDP "Substance Misuse in the Undergraduate Medical Curriculum" Accessed August 1, 2013 http://www.sgul.ac.uk/research/projects/icdp/our-work-programmes/pdfs/ substance-misuse-book.pdf

Jay, Mike High Society, Mind -altering drugs in history and culture. London: Thames and Hudson, 2010.

Jones, Deborah "One in 10 MDs has an addiction problem, physicians warned" Canadian Medical Association Journal; 149 (1) (1993): 77-78

Kay, Margaret, Mitchell, Geoffrey, Clavarino, Alexandra and Doust, Jenny. "Doctors as patients: a systematic review of doctors' health access and the barriers they experience." British Journal of General Practice 58 (552) (2008):501-508.

Lerner, Barron H. "In a Hospital Hierarchy, Speaking Up Is Hard to Do" New York Times, accessed August 1, $2013 \mathrm{http}: / /$ www.nytimes.com/2007/04/17/health/17essa.html?_r=0

Mansky, Peter A. "The Impaired physician." In Textbook of Substance Abuse Treatment, $3^{\text {rd }}$ edition edited by Marc Galanter and Herbert D. Kleber. Washington DC: American Psychiatric Press, 2011.

Marshall, E. Jane. "Doctors' health and fitness to practise: treating addicted doctors." Occupational MedicineOxford no. 58 (5) (2008):334-340.

McAuliffe, Priscilla F., Gold, Mark S., Bajpai, Lakshmikant, Merves, Michele L., Frost-Pineda, Kimberly, Pomm, Raymond, M., Goldberger, Bruce A., Melker, Richard J. and Cendan, Juan, C. "Second-hand exposure to aerosolized intravenous anesthetics propofol and fentanyl may cause sensitization and subsequent opiate addiction among anesthesiologists and surgeons." Medical Hypotheses 66 (5) (2006):874-882.

McIntosh, James and McKeganey, Neil. "Addicts' narratives of recovery from drug use: constructing a nonaddict identity" Social Science and Medicine 50 (2000): 1501-1510

McNaughton, Jane. "The humanities in medical education: context, outcomes and structures" Medical Humanities. 26 (2000):23-30

Merlo, Lisa J., and Gold, Mark. S. "Prescription opioid abuse and dependence among physicians: Hypotheses and treatment." Harvard Review of Psychiatry 16 (3) (2008):181-194.

Milligan, Barry. "Morphine-Addicted Doctors, the English Opium-Eater, and Embattled Medical Authority." Victorian Literature and Culture 33 (2) (2005):541-553.

Montgomery, Anthony. J., Bradley, Colin, Rochfort, Andree, and Panagopoulou, Efharis. "A review of selfmedication in physicians and medical students." Occupational Medicine-Oxford 61 (7) (2011):490-497.

National Institute on Drug Abuse (NIDAMED) "Medical \& Health Professionals." Accessed July 31, 2013 http:// www.drugabuse.gov/nidamed-medical-health-professionals

Notley, Caitlin., Goodair, Christine, Chaytor, Andrew, Carroll, Janine, Ghodse, Hamid and Kopelman, Peter. "Report of the substance misuse in the undergraduate medical curriculum project in England" Drugs: Education, Prevention, and Policy, accessed August 1, 2013 doi:10.3109/09687637.2013.792788 
Oyebode, F. " Illness and illness metaphor in Chekhov: Medicine and storytelling." Paper presented at the "Narrative Matters in Literature, Linguistics, Psychology and Medicine" symposium, Basel, Switzerland, April 15, 2013.

Poirier, Suzanne. "Medical education and the Embodied Physician." Literature and Medicine 25 (2) (2006): 522552

Polydorou, Soteri, Gunderson, Erik W. and Levin, Frances R. "Training Physicians to Treat Substance Use Disorders" Current Psychiatry Reports 10 (5) (2008): 399-404.

Porter, Roy and Mikulas Teich. Drugs and Narcotics in History. Cambridge: Cambridge University Press, 1998.

Ramesh, Randeep. "Doctors seek help for mental health disorders". The Guardian. Accessed August 2, 2013. http://www.theguardian.com/society/2010/jan/29/sick-medics-rehabilitation

Ramoutsaki, Ioanna. A., Askitopoulou, Helen, and Konsolaki, Eleni. "Pain relief and sedation in Roman Byzantine texts: Mandragoras officinarum, Hyoscyamos niger and Atropa belladonna." International Congress Series no. 1242 (2002): 43-50.

Rasyidi, Ernest, Wilkins, Jeffrey N. and Danovitch, Itai. "Training the next generation of providers in addiction medicine". Psychiatric Clinics of North America 5(2) (2012):461-80.

Royal College of Psychiatrists "The Mental Health of Students in Higher Education". Accessed August 1, 2013 http://www.rcpsych.ac.uk/files/pdfversion/CR166.pdf

Ritson, Bruce. "Alcohol and medical students". Medical Education, 35 (7) (2001): 622-623

Rudin, Edward, Edelson, Rachel and Servis, Mark. "Literature as an Introduction to Psychiatric Ethics." Academic Psychiatry 22(1) (1998), 41-46.

Santella, Thomas, M. Opium. New York: Infobase, 2007.

Schernhammer, Eva S., and Colditz, Graham A. "Suicide Rates Among Physicians: A Quantitative and Gender Assessment (Meta-Analysis) " American Journal of Psychiatry no. 161 (12) (2004): 2295-2302.

Stecker, Tracy "Well-being in an academic environment" Medical Education 38 (5) (2004): 465-478

Steiner, John F. "Using stories to disseminate research: the attributes of representative stories." Journal of General Internal Medicine 22 (11) (2007): 1603-1607

Stevens, Anthony. Jung. A very short introduction. Oxford: Oxford University Press, 2001

Tetzlaff, John, Collins, Gregory B., Brown, David L., Leak, Byron C., Pollock, Greg and Popa, Daniela, "A strategy to prevent substance abuse in an academic anesthesiology department." Journal of Clinical Anesthesia no. 22 (2) (2010):143-150.

Tischler, Victoria, Chopra, Arun, Nixon, Neil and McCormack, Lynne. "Loss and Tomorrow's Doctors: how the humanities can contribute to personal and professional development" International Journal of PersonCentered Medicine 1 (3) (2011), 547-552

Tischler, Victoria. "Why use the arts to teach mental health and psychiatry?" In Mental Health, Psychiatry and the Arts. A teaching handbook, edited by Victoria Tischler, 1-9. Oxford: Radcliffe, 2010.

Verghese Abraham. The Tennis Partner New York: HarperCollins, 1998.

Verghese, Abraham. "Five Best. Abraham Verghese prescribes these books on doctors' lives" Wall Street Journal, (2010). Accessed August 1, 2013. http:/online.wsj.com/article/SB10001424052748704535004575348833407184048. html

Wallace, Paul. " Medical students, drugs and alcohol: time for medical schools to take the issue seriously". Medical Education, 34 (2) (2000): 86-87

Westermeyer, Joe "Substance Use Rates Among Medical Students and Resident Physicians." JAMA: The Journal of the American Medical Association 265 (16) (1991):2110-2111. doi: 10.1001/jama.1991. 03460160088036.

Williams, William Carlos "Old Doc Rivers" in The Doctor Stories. London: Faber and Faber (1987)

Yoast, Richard A., Filstead, William J., Wilford, Bonnie B., Hayashi, Susan, Reenan, Jennifer and Epstein, Jorie "Teaching about Substance Abuse" Virtual Mentor American Medical Association Journal of Ethics 10 (1) (2008): 21-29. 